\title{
Perspective: The Experimentalist and the Problem of Turbulence in the Age of Supercomputers
}

Morteza Gharib

Professor of Aeronautics, Center for Quantitative Visualization, Graduate Aeronautical Laboratories, Pasadena, CA 91125
Due to the rising capabilities of computational fluid mechanics (CFD), the role of the experimentalist in solving the problem of turbulence has come under serious question. However, after much initial excitement by the prospect of $C F D$, the basic understanding of non-linear fluid phenomena such as turbulence still remains a grand challenge and will remain so into the unforeseable future. It appears that in order to accelerate the development of a comprehensive and practical understanding and modeling of turbulence, it is required that a constructive synergism between experiments and simulations be created. Moreover, the digital revolution has helped experimental fluid mechanics to acquire new capabilities in the whole-field flow mapping technique which enables it to efficiently interface with CFD. This new horizon is promising in its capabilities to guide, validate and actively interact in conducting reliable simulations of turbulent flows.

\section{Introduction}

Since the time of Galileo, progress in science has greatly depended on the experimentalist's capabilities to question nature by means of observation and experimentation, and by way of measuring magnitudes that could be analyzed and interrelated through mathematical formulation. It is an historical fact that every time experimental techniques have taken a leap forward, the "experimentalist" has made totally unexpected and unimagined discoveries. The history of natural science is filled with examples of experimental discoveries that have resulted in the formulation of new laws of physics by theorists. On the other hand, regardless of the way that a theory is born, ultimately, it has been the job of experimentalists to weed out the theories that work from those that don't. The dual role of experimentalists in exploration and discovery, on the one hand, and checking the newborn theories against the hard realities of the real world, on the other, creates an intricate interplay between observation and theory which is, above all, a unique demonstration of man's unintimidated but disciplined exercise of the imagination.

In recent decades, this balance between observation and theory has definitely been altered by advances in numerical methods and rising computational capabilities in the fields of engineering and physics. The remarkable advances in the computing powers of today's computers has opened the era of simulation; physical processes that can be recreated through existing laws of physics. The attractive prospects of accurately simulating the experimentally hard-to-realize problems of science at lower costs is the promise of the computational physicist or engineer. This promise is extremely appealing to the scientific and technical community in general and has been a hard one for the experimentalist to compete with. Indeed, in some branches of science and engineering where simulations can be conducted based on firm and well-behaved mathematical models, the role of the experimentalist has grown weaker. However, the role of experiments in weeding out the simulations that work from those that don't continues to be essential in all branches of science and technology. Nonetheless, the concept of exploration and discovery through simulation, rather than experimentation, has most threatened the role of the experimentalist as discoverer.

Contributed by the Fluids Engineering Division for publication in the JourNal OF FLUIDS ENGINEERING. Manuscript received by the Fluids Engineering Division September 12, 1995; revised manuscript received February 16, 1996. Associate Technical Editor: D. P. Telionis.
Among many fields of science that have faced this threat, fluid mechanics has suffered the greatest impact. In the past 100 years, from the primitive wind tunnel studies of the Wright brothers to the large scale wind tunnels of national laboratories, and from the desktop experiments of G. I. Taylor to sophisticated oceanographic laboratory platforms, experiments have been the only viable source of knowledge for basic or industrial fluid mechanics research. The rapid advances in computational fluid dynamics in the 70 s and early 80 s have been responsible for the creation of the narrow view that experimental facilities (for example, wind tunnels) would eventually be replaced by their digital counterparts. Such views, which were based on projections of dramatic increases in the performance of computers, reflected a lack of appreciation for the non-linear and multidimensional nature of many of today's unresolved grand scientific challenges, of which a majority happen to reside in the field of fluid mechanics. In the middle of the broad range of flow problems that challenge today's scientists stands the greatest puzzle of classical physics-turbulence.

\section{The Grand Challenge}

Fluid turbulence, in its many forms, shapes and degrees, from the grandest reaches of space to the sand and sea, is the elemental dynamic form. Its appearance in the atmosphere, in plasma, in fluid interfaces or in multi-phase flows is a manifestation of the convective and nonlinear nature of physical laws that govern the flow of fluids. The problem of understanding turbulence, in order to model and predict its mixing and transport properties or the prediction of flow around and inside geometries, is the grand challenge of this and quite possibly the next century. Turbulence has remained the last frontier of twentieth century science. For all the efforts of the great minds of science, the problem of turbulence has remained empirical.

The history of fluid mechanics would certainly credit the foundation of our current understanding of turbulence to the bold and pioneering work of scientists such as Osborn W. Reynolds, G. I. Taylor, Ludwig Prandtl, and Theodore von Kàrmàn who were products of a scientific culture that exercised the aforementioned intricate interplay between experiments and theory. Since their milestone work, many subsequent theoretical and modeling efforts to represent the physical process of turbulence have been pursued. Ultimately, these theories and models had to face the practical demands of industry where predictive capabilities were required. The rise of computational fluid dy- 
namics was a natural answer to this demand for which experimentalists and theorists had not yet provided a viable solution.

After much justifiable initial excitement by the prospects of computational fluid dynamics, the basic understanding of fluid turbulence in its various forms (plasma, climatology, hydro, and aerodynamics) still remains a challenge to the theorist, experimentalist and numerical analyst alike. The colossal nature of this problem requires the development of novel approaches based on the synergism that experimentalists, numerists and theorists can create. This synergism should provide a higher level of validation for turbulence models by reaching deeper levels of understanding for the physics of turbulence. In order to construct this synergism, one needs to appreciate the nature of the problem of turbulence and identify the issues that currently slow the progress toward achieving a comprehensive understanding of this problem. In doing so, I have tried to express my opinion and understanding of these issues from the point of view of an experimentalist. In many cases, the ideas expressed in this paper are far from novel in their essence, but they reflect the means shared by many traditional and "contemporary" experimentalists. The particular examples cited in this paper only partially represent the state-of-the-art and are by no means the only ones. For the sake of brevity, I have tried to avoid combing the literature for every possible similar work, which I hope will not lessen the credibility of the ideas presented in this paper.

\section{Some Insights}

Many fluid mechanical problems of scientific and technological importance exhibit complex, unsteady and multi-dimensional dynamics. Large-scale turbulent separated flows, turbulent mixing, combustion and geostrophic flows provide a few unsteady examples. Above all, the three-dimensionality of these flows poses the greatest difficulty in the simulation or measurements of these flows. In this respect, some scaling arguments will help us to appreciate what it takes to "resolve" turbulence through experimentation and simulation.

We would like to consider turbulence as an ensemble of interacting eddies, where energy is provided by the large eddies and lost by the viscous action of the fluid on the smallest eddies. Based in this scenario and dimensional analysis, Kolmogorov's scaling arguments for turbulence (1941) suggest,

$$
\frac{\delta_{\max }}{\delta_{\min }} \sim \operatorname{Re}^{3 / 4}
$$

where $\delta_{\max }$ is the nominal size of the large energy-containing eddies in a shear flow and $\delta_{\min }$ is the size of the smallest existing eddies (known as Kolmogorov eddies) and $\mathrm{Re}$ is the Reynolds number (i.e., $\operatorname{Re}=\delta_{\max } U / v$ ) based on the $\delta_{\max }$ and the typical velocity of large eddies (i.e., $U$ ). If we assume that $\delta_{\min }$ represents the minimum spatial resolution, then the above ratio will become an indication of the number of grid points that the computation or measurements will be required to have in order to describe the entire dynamic range of scales (Frisch and Orszag, 1990). In $3-D$, this ratio would be proportional to $\left(\mathrm{Re}^{3 / 4}\right)^{3}$.

The minimum temporal resolution can be obtained by dividing the smallest spatial scale $\left(\delta_{\min }\right)$ by the convective velocity of large eddies $(U)$

$$
\tau_{\min }=\frac{\delta_{\min }}{U} \sim \frac{\delta_{\max }}{U} \operatorname{Re}^{-3 / 4} .
$$

Again, the ratio of the largest to smallest temporal scales can be expressed as

$$
\frac{\delta_{\max } / U}{\tau_{\min }} \sim \operatorname{Re}^{3 / 4},
$$

which also indicates the number of required time steps (Karnia- dakis and Orszag, 1990). Therefore, the minimum number of points that ultimately need to be obtained is proportional to

$$
N_{\text {total }} \equiv\left(\operatorname{Re}^{3 / 4}\right)^{3} \cdot\left(\operatorname{Re}^{3 / 4}\right)=\operatorname{Re}^{3} .
$$

This estimate is quite conservative since the minimum number of required grid points in time and space, in order to reliably resolve the spectral properties of turbulence, should follow the Nyquist criteria for space and time, in that it should be sixteen times higher. This requirement for the minimum number of points in measurements or computations, in order to describe the flow, is rather depressing since every order of magnitude increase in the flow Reynolds number requires a three orders of magnitude increase in the number of required computational/ experimental grid points.

To scale the problem, consider the fact that, for the onset of fully developed turbulence in shear flows, a minimum Reynolds number of $10^{4}$ is usually required (Dimotakis, 1993). At the outset, for transport airliners or atmospheric flows, the Reynolds number will be in the range of $10^{8}$ to $10^{12}$, respectively. The sobering resolution requirement for performing practical measurements or simulations of turbulent flows in this range demands computing powers and diagnostic tools beyond the capabilities of standard available technology.

\section{Computing the Turbulence}

What might have been called a supercomputer in the $1980 \mathrm{~s}$ (to distinguish it from the standards of the industry at that time) will be hard-pressed to compete with some of the hand-held PC's of the 1990s. Therefore, the term supercomputer is a relative but attractive name and, therefore, should be judged based on what it achieves. This term has been passed on from sequential (von Neumann) machines to today's parallel processors with giga $\left(10^{9}\right)$ and, very soon, tera $\left(10^{12}\right)$ flops (floating-point operations per second) capabilities.

Application of paralleled codes on massively parallel computers with efficient networking and a large memory capacity are becoming the methods of choice for simulating turbulent flows. In the background of the ever-increasing power of supercomputers, there exists a whole spectrum of methodologies that can be used to compute turbulent flows - some with short term objectives and some with a look toward a comprehensive approach to the turbulence problem.

Ideally, one should be able to compute an entire flow field by solving the 3-D unsteady Navier-Stokes equations. This approach, which is known as direct numerical simulation or DNS, should (within the numerical approximation) render the full range of turbulent motions as well as transport properties of the flow. DNS is an alternative to experimental realization in the laboratory with all the attractive aspects of exploration and discovery which have been unique to experiments. However, the restriction caused by the aforementioned resolution requirements inhibits the practical application of DNS in simulating turbulent flows with a Reynolds number in excess of $10^{4}$. In this respect, considering the current state-of-the-art in computing and the most optimistic prediction for its expansion, DNS applications will be confined to turbulent flows involving simple geometries at Reynolds numbers below $10^{4}$ for many years to come. For example, using an efficient DNS code, a typical calculation of 100 cycles of vortex shedding from a circular cylinder at a Reynolds number of 1000 (Henderson and Karniadakis, 1995; see also Appendix ) takes up to 400 hours to generate on a parallel supercomputer with a speed of 0.5 giga flops.

Despite the current limitations of direct simulations, DNS has proved that, when it is used appropriately, it is an unsurpassed method for understanding the physics of flow, interacting with experiments, or guiding modeling efforts. We will discuss the unique opportunities that DNS provides in the next section. However, the practical needs of industry demand intermediate 
solutions for high Reynolds number flow problems in the form of predictive models.

At the heart of practical technological problems is the need for predicting turbulent fluxes of momentum and passive scalars such as species and temperature. Depending on the nature of properties in demand, various approximation schemes have been developed that do not involve omission of viscous or turbulent stress terms. All of these methods represent some kind of averaging or filtering operations on the $\mathrm{N}-\mathrm{S}$ equations. In its simplest form, the averaging or filtering of N-S takes the following form:

$\rho\left(\frac{\partial \bar{u}_{i}}{\partial t}+\bar{u}_{j} \frac{\partial \bar{u}_{i}}{\partial x_{j}}\right)=-\frac{\partial \bar{p}}{\partial x_{i}}+\frac{\partial}{\partial x_{j}}\left(\bar{\tau}_{i j}+T_{i j}\right)$,

where

$$
\begin{aligned}
u_{i} & \equiv \text { velocity vector } \\
p & \equiv \text { pressure } \\
\tau_{i j} \text { (viscous stress) } & \equiv \mu\left[\left(\partial \bar{u}_{i} / \partial x_{j}\right)+\left(\partial \bar{u}_{j} / \partial x_{i}\right)\right] \\
\rho & \equiv \text { density (constant) }
\end{aligned}
$$

\section{$T_{i j}$ (Reynolds stress tensor) $\equiv-\rho \overline{u_{i}^{\prime} u_{j}^{\prime}}$}

The overbar represents the filtering or averaging process in the form of spatial, temporal or ensemble averaging. On one extreme of the filtering processes stands the Reynolds (1985) scheme which results in Reynolds averaged N-S or RANS equations where the entire time dependent parameters are averaged based on the assumption of statistically steady turbulence. In general, the averaging or filtering introduces correlations $\left(-\rho \bar{u}_{i}^{\prime} u_{j}^{\prime}\right)$ between unresolved fluctuating velocities which act as stresses $\left(T_{i j}=-\rho \overline{u_{i}^{\prime} u_{j}^{\prime}}\right)$ on the resolved motions. Equation (1) is not closed until a model is constructed that can relate the Reynolds stress $\left(T_{i j}\right)$ to the mean velocities $\left(\overline{u_{i}}\right)$.

Through Boussinesq's hypothesis, the Reynolds stresses can be related to the gradients of averaged or filtered velocities (strain rate tensor) through the following eddy-viscosity relation for incompressible flows:

$$
T_{i j}=\mu_{T}\left(\frac{\partial \bar{u}_{i}}{\partial x_{j}}+\frac{\partial \bar{u}_{j}}{\partial x_{i}}\right) .
$$

The variation of the eddy viscosity $\mu_{T}$ over space and time must be obtained through turbulence modeling. The first models for closure were proposed by Prandtl (see Hinze, 1959) and is known as the mixing length theory. For two-dimensional flows, it suggests

$$
v_{T}=l_{\mathrm{o}}^{2}\left|\frac{d u}{d y}\right| \quad \text { or } \quad v_{T}=l_{1} \Delta u
$$

$l_{1}$ is the shear layer thickness while $l_{0}$ is the mixing length. For the near wall region of turbulent boundary layers, the mixing length is related to the transverse coordinate $(y)$ as $l_{0}=K y$ where $K$ is the von Kàrmàn constant. This is a one-dimensional model that is mainly applicable to turbulent boundary layers. Validation studies of RANS codes of this type are mainly limited to two-dimensional flows. A particular three-dimensional version of the Prandtl model is the Baldwin-Lomax Model (Baldwin and Lomax, 1978), which has been very popular for RANS models in aerodynamics. It takes the following form:

$$
v_{T}=l_{0}^{2}\left(\overline{\omega_{i} \omega_{i}}\right)^{1 / 2}
$$

where $\bar{\omega}=\nabla \times \bar{u}$ is the mean vorticity vector (Speziale, 1990).

\footnotetext{
In relation (2), we have neglected the normal stresses.
}

The predictive power of this type of RANS model is limited since the turbulent length scale $\left(l_{o}\right)$ should be independently provided for every specific application. One can avoid direct modeling of turbulent shear stresses by using a corresponding transport equation for the Reynolds stress itself, which results in the generation of additional higher order correlations to be modeled. The modeling of this higher order correlation is a central challenge to the statistical theory of turbulence. The application of RANS codes to massively 3D and transient flows has been limited to simple geometries. To obtain the drag coefficient or the pressure distribution, one needs to accurately model the separation regions where the dynamics have a profound effect on the separation points and thus on the base pressure distribution. In defining the averaging schemes, one can design filters that preserve certain dominant frequencies and average out stochastical turbulent motions altogether. This is an unsteady version of RANS codes whose application again requires explicit experimental input (Rodi, 1993).

Roshko (1992) points out that "one impact of [the original] RANS methods and the statistical theory was to tend to encourage a view of 'fully turbulent' flow as too complicated and disorganized to contain structural features that could be usefully incorporated into any model." The discovery of large vortical structures in boundary layers (Kline et al., 1967) and high Reynolds number mixing layers of Brown and Roshko (1974) has certainly changed this view. The impetus for an alternative method that stands between the two extreme approaches of DNS and RANS codes stems from these discoveries. Large eddy simulation methods tend to benefit from the fact that, for high Reynolds number flows, the large eddies are responsible for the transport of momentum and energy in recirculating regions, in free shear flows and in setting up the large scale pressure fields. In large eddy simulations, the behavior of the large scale motion of turbulence is simulated through conventional DNS codes, while modeling is used for the smaller unresolved scales. The fundamental aspect of LES is in the projection operation or filtering of the N-S equation. Similar to the RANS approach, the resulting truncated $\mathrm{N}-\mathrm{S}$ equation lacks closure because of the stress-like reflection of eddies that fail to pass the filter on those that do (Ferziger, 1983; Rogallo and Moin, 1984). This stress-like reflection, which is called subgrid-scale (SGS) Reynolds stress, must be related to the large scale eddies in order for the scheme to work.

The Smagorinsky model, which is a 3D version of the eddy viscosity model, has been used to tackle the closure issue. In this model the SGS Reynolds stress tensor $\overline{u_{i}^{\prime} u_{j}^{\prime}}$ is assumed to be related to the corresponding components of the strain rate tensor of the large scalar field, $\left(\bar{S}_{i j}\right)$, through

$$
\overline{u_{i}^{\prime} u_{j}^{\prime}}=v_{T} \bar{S}_{i j}=\frac{1}{2} v_{T}\left(\frac{\partial \bar{u}_{i}}{\partial x_{j}}+\frac{\partial \bar{u}_{j}}{\partial x_{i}}\right)
$$

where $v_{T}$ is the SGS eddy viscosity given by

$$
v_{T}=C_{s}\left(\bar{S}_{i j} \bar{S}_{i j}\right)^{1 / 2} \bar{\Delta}
$$

where $\bar{\Delta}$ is the corresponding length scale associated with the low pass filter and $C_{s}$ is a constant parameter.

Many variations and modifications of the Smagorinsky model (e.g., dynamic SGS models, Germano et al., 1991) have been developed and used to attain the proper reflection of the unresolved scales on the resolved large eddies. The improper modeling of small scales results in excessive dissipation and can have catastrophic results in terms of the behavior of large scales.

The main shortcoming of the RANS and LES approaches is the fact that turbulent time and length scales are not universal and their modeling requires empirical information provided by experiments. A true modeling of the small scales in fully developed turbulent flows require experimental construction of strain rate and turbulent stress tensors. This is an exceedingly chal- 
lenging task, since the current available experimental techniques can hardly render such information for three-dimensional flows.

The last method that we must mention, and what Ferziger (1993) calls the chief competition of the LES technique for the simulation of turbulent separated flows, is the discrete vortex method (Leonard, 1985; Sarpkaya, 1988). Excellent results, in terms of the flow pattern and of flow quantities at very large Reynolds numbers, can be obtained for 2-D geometries by this technique. Application of this technique to 3-D complex flows is the subject of much current research.

\section{Probing the Turbulence}

Single point measurement techniques such as hot wire and laser Doppler velocimetry (LDV) have been widely used for turbulence studies. Hot wire anemometry and LDV had been perfected and are still in use for many fluid measurements and control applications, while remaining useful for obtaining various quantities at a single point or array of points as a function of time. Still, the Eulerian nature of the information obtained by these devices yields insufficient information for an adequate description of turbulence. The discovery of large vortical structures at high Reynolds number flows through flow visualization (Brown \& Roshko) proved that single point probes can miss the big picture altogether.

Naturally, flow visualization, benefiting from a remarkable function of the human brain in recognizing moving patterns, has helped the observers of nature-from Leonardo Da Vinci to modern vortex chasers - to appreciate the Lagrangian nature of turbulent flows. In science and technology, wherever adequate visualization has been part of the creative process, it has yielded new insights into complex phenomena and has provided better descriptions and predictive models. Examples range from Osborn Reynolds' description of turbulence to the observation of large vortical structures by Brown and Roshko. From the mixing of dye patterns to patterns generated by the pathlines of small suspended particles, the rendered information has been a remarkable source of knowledge. Obviously, the global nature of this information has invited many attempts to develop quantitative methods for flow visualization. A major drawback in doing so has been the lack of advanced imaging, acquisition and subsequent processing hardware and software technology. It is interesting that the same digital revolution that converted the primitive abacus machines to today's supercomputers has also helped experimenters to develop some of the most amazing means of quantitative imaging. In other words, this revolution has helped to develop the new art and science of quantitative visualization.

\section{Quantitative Flow Visualization}

Quantitative flow visualization has many roots and has taken several approaches. The advent of digital image processing has made it possible to practically extract useful information from every kind of flow image. In a direct approach, the image intensity or color (wavelength or frequency) can be used as an indication of concentration, density and temperature fields or of gradients of these scalar fields in the flow (Merzkirch, 1987). These effects can be part of the inherent dynamics of the flow (e.g., gradients of density are used in shadowgraph and Schlieren techniques) or generated through the introduction of optically passive or activate dye agents (fluorescent tracers, liquid crystals) or various molecular tagging schemes.

In general, the optical flow or the motion of intensity fields can be obtained through time sequenced images (Singh, 1991). For example, the motion of patterns generated by dye, clouds or particles can be used to obtain such a time sequence. The main problem with using a continuous-intensity pattern, generated by scalar fields (e.g., dye patterns), is that it must be fully resolved (space/time) and contain variations of intensity at all scales before mean and turbulent velocity information can be obtained (Pearlstein, 1995). In this respect, the discrete nature of images generated by seeding particles has made particle tracking the method of choice for whole field velocimetry. The technique recovers the instantaneous two- or three-dimensional velocity vector field from multiple images of a particle field within a plane or volumetric slab of a seeded flow, which is illuminated by a laser light sheet. Various methods such as individual tracking of particles or statistical techniques can be used to obtain the displacement information and subsequently the velocity information. The spatial resolution of this method depends on the number density of the particles. A major drawback in using particle tracking techniques has been the unacceptable degree of manual work that was required to obtain the velocity field from a large number of traces or particle images. Digital imaging techniques have helped to make particle tracking less laborious (Gharib and Willert, 1989). However, because of the errors involved with identifying the particle pairs in high particle-density images, the design of automatic particle tracking methods for three-dimensional flows has been especially challenging. Therefore, applications of the automatic particle tracking methods have been limited to low particle density images.

In contrast to the tracking of individual particles, the particle image velocimetry (PIV) technique follows a group of particles through statistical correlation of sampled windows of the image field (Adrian, 1991). This scheme removes the problem of identifying individual particles which is often associated with tedious operations and large errors in the detection of particle pairs. In terms of the spatial resolution, the obtained velocity at each window represents the average velocity of the group of particles within the window. The interrogating window in PIV is the equivalent of the grid cell in CFD. Development of the video-based digital version of PIV, known as DPIV (Willert and Gharib, 1991; Westerweel 1993), resulted directly from advances in charge coupled device (CCD) technology and fast, computer-based, image processing systems. In terms of overall steps involved, DPIV is much faster than photographic PIV and particle tracking methods. The spatial resolution of the DPIV technique is decided by the resolution of the CCD array which is usually less than photographic films. However, this shortcoming is being reduced by the continuing improvement in high resolution/high speed CCD technology.

The capability of whole field measurement techniques in providing velocity vector or scalar field information in a format compatible to CFD calculations has made a major impact in defining common ground for designing new approaches toward resolving the turbulent flow problem. Figure 1 presents an example of companion DPIV (Gharib and Weigand, 1995) and DNS (Zhang and Yue, 1995) simulations of the interaction of a vortex ring (Reynolds number of 1000 ) with a free surface. The velocity vector fields are shown at the surface and in the symmetry plane of the vortex. The DNS simulation was carried out using non-linear free-surface boundary conditions. For DPIV, two simultaneous laser sheets and two simultaneous video-cameras were used to map the flow. Figure $1(c)$ shows temporal evolution of the vorticity field at the free surface and the symmetry plane. A single flow parameter such as circulation has been used to draw a quantitative comparison between the two approaches. Qualitative and quantitative agreements in the domains of time and space serve to confidently use the CFD results to explore other flow parameters that are not obtainable through experiments. Such a comparison could not be obtained by using methods such as LDV or hot wire anemometry that do not address the global Lagrangian and the temporal nature of this flow.

DPIV can be utilized to obtain three components of the velocity field (Raffel et al., 1995). However, this extension of DPIV is limited to a few planes and cannot address the full dimensionality of turbulent flows with the current video technology. Holo- 

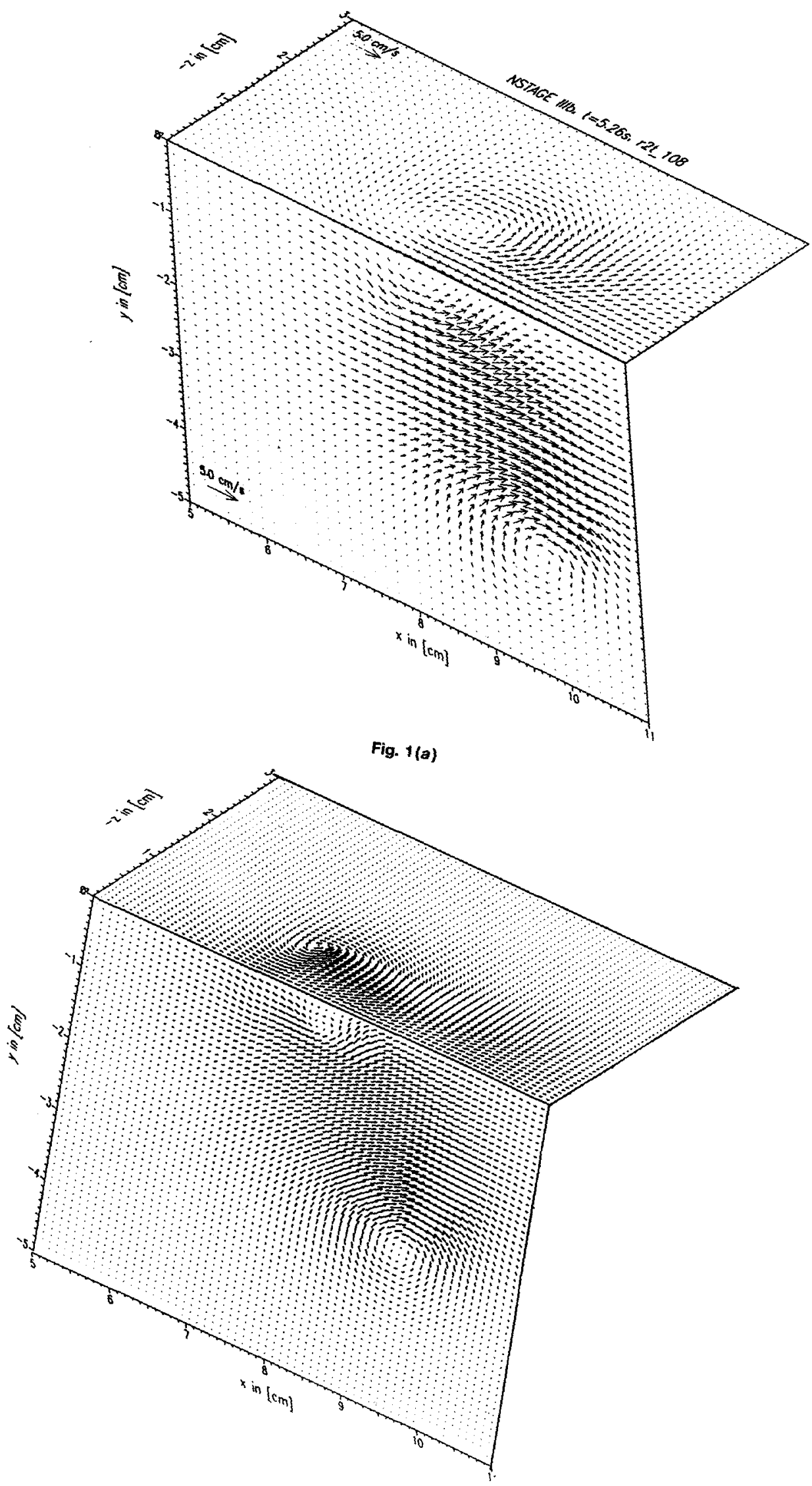

Fig. $1(b)$ 


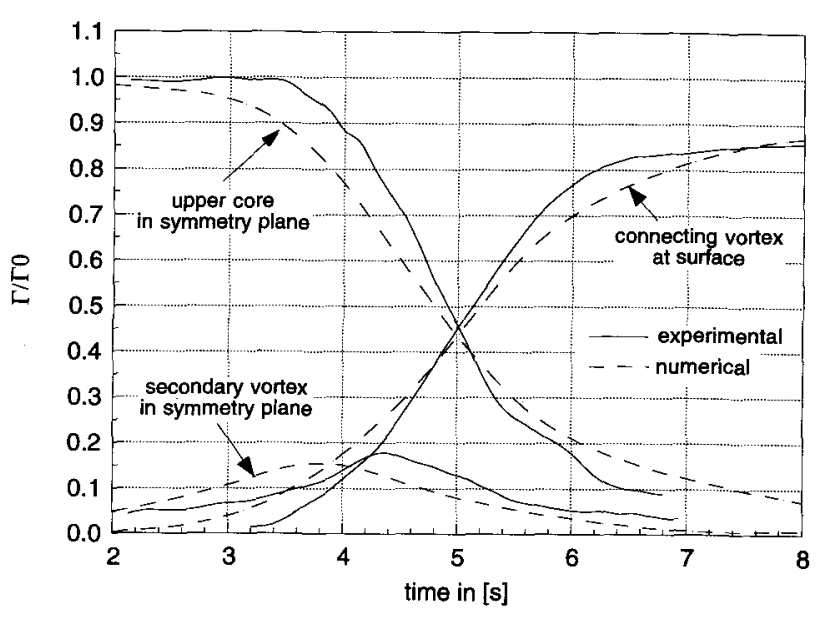

Fig. 1 (c)

Fig. 1 The velocity vector field obtained by (a) DPIV (Gharib and Weigand, 1995) and (b) DNS (Zhang and Yue, 1995)

graphic PIV techniques are more suitable for obtaining threedimensional distribution of the velocity vector field (Barnhart et al., 1994). Figure 2 depicts such a three-dimensional mapping of velocity vector field in a fully developed turbulent channel flow. The photographic nature of holographic PIV techniques limits their ability to address the temporal dynamics of turbulent flows. Recent advances in three-dimensional video-based particle tracking techniques have removed some of these shortcomings (Kasagi and Sata, 1992).
Besides DPIV, quantitative scalar imaging techniques have also been developed for concentration measurements (Koochesfahani and Dimotakis, 1986), for temperature (Dabiri and Gharib, 1991), and for surface elevation and slope (Zhang and Cox, 1994; Zhang, 1994). In the latter, the surface slopes can be directly obtained from a novel color-coding technique; an example of its use to study free-surface turbulence generated by an underwater jet is given in Fig. 3. Each color in this picture is representative of a certain slope which can be converted to the surface elevation through a spatial integration scheme.

The aforementioned examples from the author's group and others represent various whole field quantitative flow visualization methods that hold the promise of providing new dimensions in measurements and their interaction with CFD.

\section{Defining Common Ground}

It would be greatly deceiving to attribute the shortcomings of simulations to the limitations of computational power or numerical issues (Rizzi and Engquest, 1987; Leschziner, 1993). There are many other issues that severely challenge various computational approaches to solving turbulent flow problems including:

1. Steady and unsteady boundary conditions (common to all types of CFD methods)

2. Complex 3-D geometries (common to Euler methods, RANS, LES, vortex methods)

3. Multi-phase flows (common to all types of CFD methods )

4. Coupled-fields (common to all types of CFD methods)

5. Compressibility (common to all types of CFD methods)

\section{HPIV}

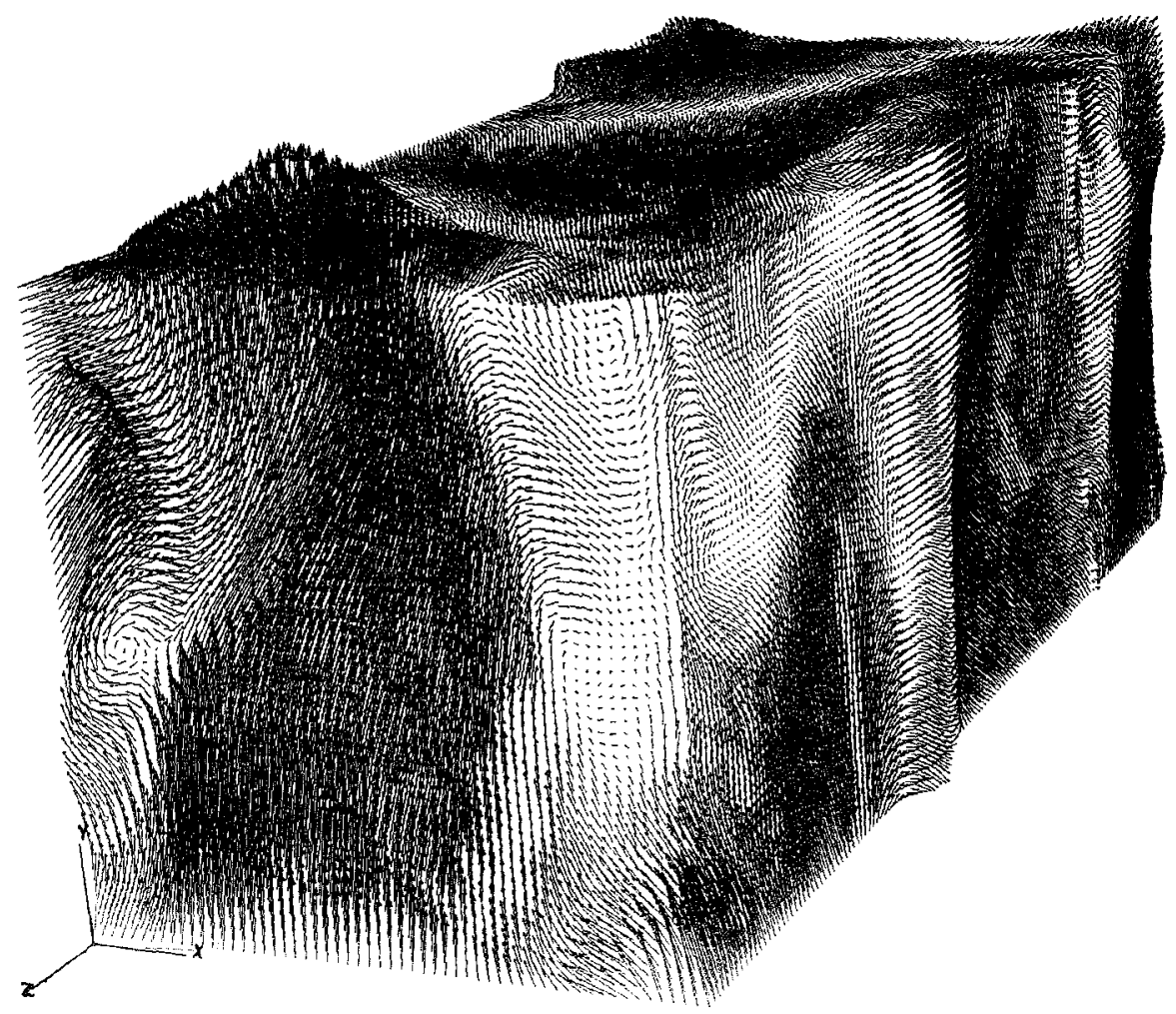

Fig. 2 Three-dimensional velocity vector field obtained by a phase-conjugated holographic PIV technique (Barnhart, 1994) 


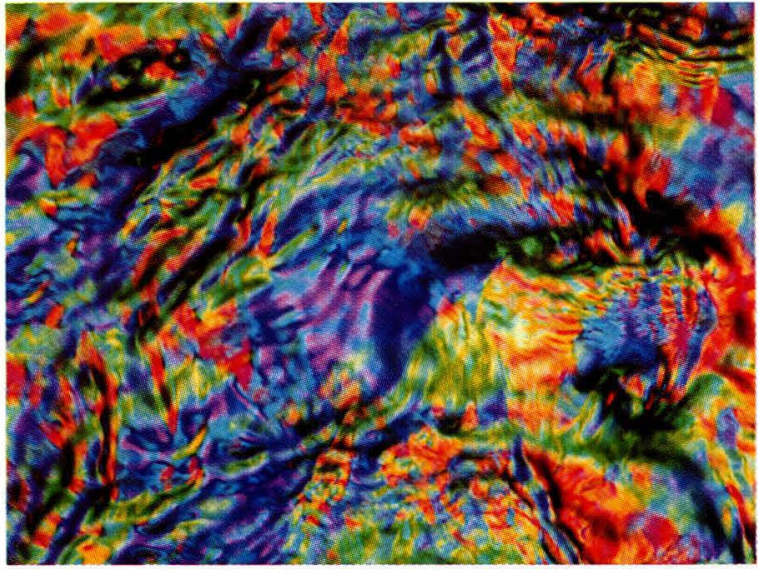

Fig. 3 Free-surface color slope mapping of a near surface turbulent jet (Zhang et al., 1994)

In this respect, the interaction between experiments and CFD can take place at two levels:

1. Low and Medium Reynolds Number. For straight validation purposes; such as checking on the two-dimensionality of the flow, geometry definition, and velocity and vorticity field comparisons; quantitative flow visualization methods offer a unique opportunity. In our second example shown in Fig. 4 simulation of vortex shedding from a circular cylinder using DNS (Henderson 1994) is compared with its DPIV counterpart.
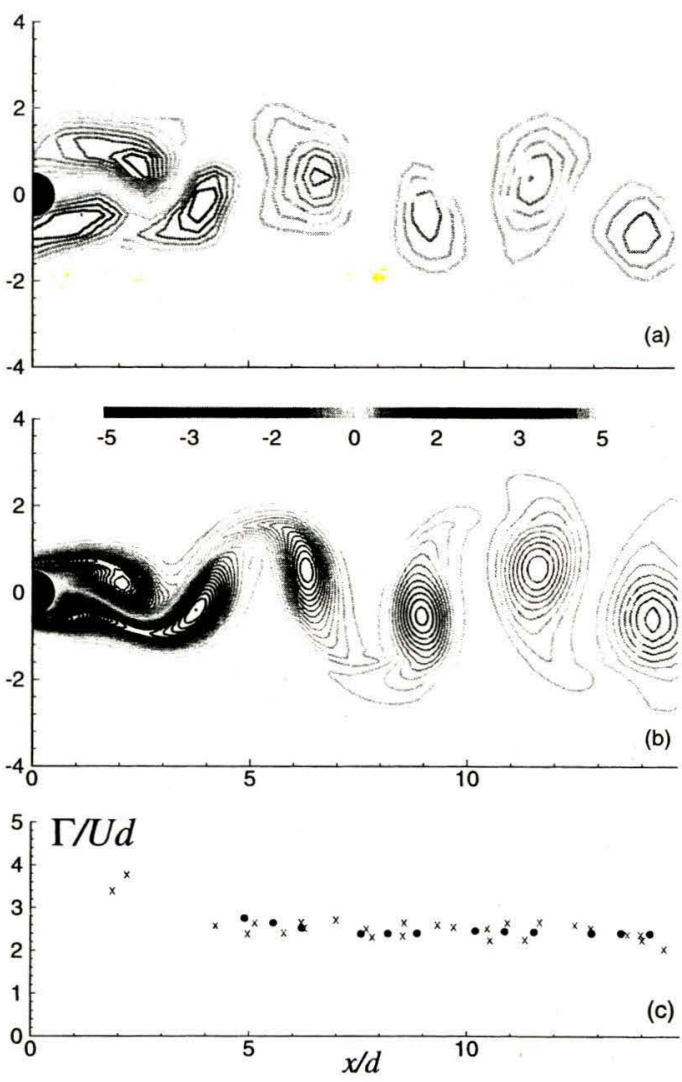

Fig. 4 Vorticity in the wake of a circular cylinder, $R e=100:(a)$ DPIV measurements, (b) 2-D numerical simulations, (c) computed values of the circulation of wake vorticies from $(\times)$ experiments and $(\bullet)$ simulations. This example is typical of the excellent qualitative (vortex size and shape) and quantitative (circulation) agreement now possible for unsteady two- and three-dimensional flows.

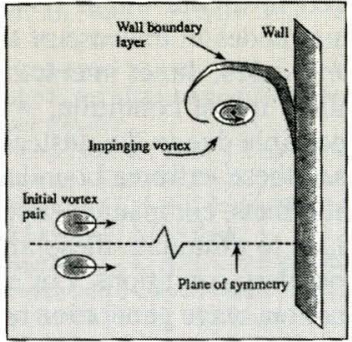

Schematics of the initial vortex pair and its interaction with the wall.

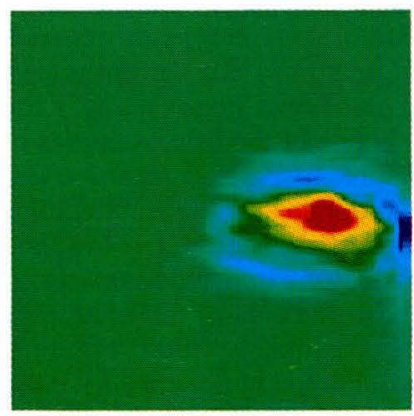

DPIV results

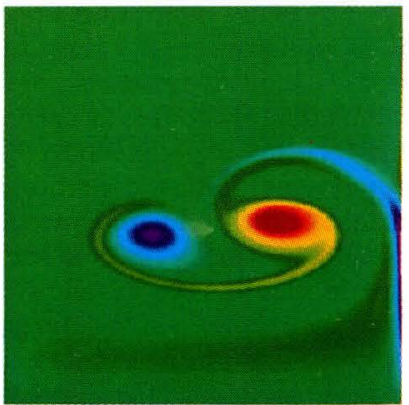

DNS simulation with Gaussian vorticity distribution for the initial vortex.

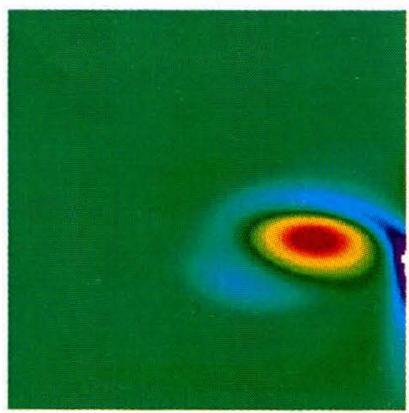

DNS with vorticity distribution from DPIV for the initial vortex.
Fig. 5 A comparative study of DNS simulation based on initial conditions from a model or DPIV vorticity distribution (Liepmann and Dommermuth 1991; Fabris et al., 1995)

Experiments are essential in resolving modeling issues, such as boundary conditions, where modeling errors can produce errors in the solution of order one. The problem of defining the proper in-flow (upstream) condition and the determination of the dimensions of the computational box (blockage effect and correlation lengths ) can easily benefit from appropriate laboratory simulation of the flow condition. One of the most important features of DPIV is the compatibility of its format with that of the CFD method. In-flow conditions or initial flow properties can be used directly as input to initiate DNS or LES. This approach removes unrealistic in-flow conditions which often result in dubious flow solutions. In Fig. 5( $a),(b),(c)$, and $(d)$, we present the interaction of a vortex pair with a solid boundary obtained by DPIV and DNS. Figure $5(b)$ shows the DNS simulation initiated by a Gaussian vorticity distribution for the approaching vortex. Figure $5(c)$ depicts the DPIV results. Figure $5(b)$ shows that the simulation does not generate the correct flow field if the initial experimental conditions were not used. Using initial vorticity fields from the DPIV measurement, Figure $5(d)$ shows a much better agreement with experiments. It is interesting to note that without such novel experiments, the whole validity of the DNS code used for this simulation might have ended in doubt for a wrong reason (Liepmann and Dommermuth, 1991; Fabris et al., 1995). Also, it has to be mentioned that such experiments open new opportunities for interactive computational/experimental methods to be developed for thermofluid flows (Humphrey et al., 1991).

There are two other types of boundary conditions that are especially troublesome; namely, boundaries that involve fluid that leaves the computational domain (out-flow condition), and unsteady moving boundaries such as flexible cables (Newman and Karniadakis, 1995) or free surfaces (Dommermuth, 1993). For simulations, these boundary conditions are part of the solution to the problem and need to be defined in transit. Proper resolutions of these problems are important for situations involving free surfaces as boundary conditions. Exit conditions 
are especially interesting since they can create feedback problems upstream in the form of altered upstream initial conditions-acoustic resonance or sloshing modes in the case of the simulation of the free surface. In these cases, direct interaction between the experimentally determined initial condition, wall condition or in-flow condition is impossible due to the unsteady nature of the problem. However, since these extreme boundary conditions usually result in additional effects, companion experiments can be used to tune simulations to eliminate these artifacts and obtain realistic dynamic boundary conditions. For example, studies of free surface deformation, wave generation and wave reflection off the computational boundaries can be studied under realistic experimental conditions. Techniques such as the surface mapping technique can become very valuable in resolving these types of boundary problems.

DNS simulations at low to medium range Reynolds numbers ( $2000<\operatorname{Re}<5000$ ) have been helpful to our current understanding of turbulent channel and pipe flows. For example, Kim, Moin and Moser's paper (1987) is one of the best examples of such an achievement, where a comprehensive flow database was used for analysis and providing guidelines for higher Reynolds number experiments. In this respect, Eggels et al. (1994), and Westerweel et al. (1995) have conducted extensive comparisons of DNS, LDV, DPIV and PIV measurements of turbulent pipe flows at Reynolds number of approximately 5000. Figure 6 shows the power spectra of the axial velocity obtained by these four methods. It is interesting to note that DNS results indicate the poor resolution of the selected DPIV and PIV parameters in high wave number ranges. This problem can be solved by reducing the imaging area. On the other hand, at low wave numbers, the short period of DNS calculations results in an underestimation of the power spectra. LDV appears to reflect the flow properties correctly at high and low wave number ranges. However, the $\mathrm{LDV}$ information is one-dimensional (only in time) and fails to provide the spatial correlation information which is essential for RANS and LES simulations. DNS can be quite valuable in providing resolution analysis for LES and for experiments (Jimenez, 1994) or guiding experiments in resolving fine flow structures (Visbal, 1994; Lin and Rockwell, 1995).

As Reynolds number increases, a turbulent flow involves either high speed or large scale or both. The current limitation of video technology in providing high speed imaging limits the capabilities of techniques such as DPIV in providing timeresolved temporal behavior of the flow. Spatial resolution can also become an issue, but-at the expense of the dynamic range - this problem can be avoided. For this range, CCDbased whole-field measurement techniques can be used to construct reliable mean and fluctuating flow statistics. For example, length scale correlation of energy-containing eddies, obtained through PIV, can be used to truncate the DNS and computational domain (Le Penven et al., 1985). Such studies would be important in constructing SGS models for large eddy simulation of turbulent flows in medium range Reynolds numbers.

2. High Reynolds Numbers. LES, while very promising for high Reynolds number applications, can become quite expensive when, for example, the wall layers need to be resolved. Simple turbulent boundary layer flow approximations, such as "law of the wall" (Murakami et al., 1985), can be used as the boundary condition to alleviate the fine grid requirements. For more complex 3-D flows, such as junction flows or free surfaces, such approximations do not exist; therefore, SGS modeling cannot be avoided. On the other hand, the majority of SGS models, such as the Smagorinsky model, are based on the assumption of isotropic flow conditions in the wall region. While the Smagorinsky model, based on the isotropy assumption, works well for wall bounded flows (Saddoughi, 1994), its reliable application to more complex flows; such as massively separated flows, jets, wakes, or flows near free surfaces (Sarpkaya et al., 1994); remains to be verified. Experimental techniques with multidimensional capabilities should be used to address the question of anisotropic turbulent viscosity for complex flows through measurements of Reynolds stresses and strain rate fields. In the meantime, methods such as DPIV and LDV can be used to measure dissipation rates which can be used directly in LES models. It is imperative to the problem of turbulence that such interfaces between simulations and scalar field measurements be developed. For example, spectral behavior of free-surface fluctuation such as the one shown in Fig. 3 will be essential in SGS modeling and also for estimating the dissipation rate in the near-surface region.

Regardless of whether a viable solution can be found for SGS modeling, the LES for high Reynolds numbers would still be expensive since it requires the computation and storage of a large quantity of data for the purpose of extracting statistics. In this regard, RANS approaches are the only available methods for obtaining detailed flow information for very high Reynolds number flows. Examples include ship hydrodynamics (Tahara and Stern, 1994) and wind engineering (Rodi 1993). Information that has been obtained by single point measurement in terms of mean quantities and correlations has been instrumental in the development and application of the RANS method to boundary layer problems. However, further development of RANS has been restricted by the lack of knowledge regarding spatial correlation functions for the cases where separated flows or free surfaces are involved. According to the statistical theory of turbulence, spatial correlations provide useful knowledge (Batchelor, 1953; Lesieur, 1986) about the structure of turbulence and can be used in RANS methods. Also, this information can be used to design mathematical models of physical vortical turbulence for RANS methods (Lundgren, 1982; Pullin and Saffman, 1993). Development of such models, based on information that can be obtained from whole field velocimetry techniques, is essential for the further development of classical modeling.

In order to give RANS models predictive capabilities, the need for empirical constants must be removed. One exciting possibility is to incorporate the underlying, driving instability of the flow, based on mean velocities, to obtain a prediction of Reynolds stresses without using any empirical constants (Liou and Morris, 1992). The future development of this method would depend on the construction of amplitude equations that can correctly represent instability characteristics of various shear flows. It is essential that the mean velocity profile and its spatial evolution be incorporated into these amplitude equations. Quantitative whole field velocimetry techniques can readily provide global mean flow information at high Reynolds numbers beneficial to this technique.

\section{Conclusion}

In the last two decades, considerable national, human and monetary resources have been redirected to the development of CFD. Despite the phenomenal increase in computing power, the promise of economical, realistic simulation of turbulent flows in practical Reynolds number ranges $\left(>10^{5}\right.$ ) has not been realized. In this respect, the confluence between computational fluid dynamics and experiments, beyond its traditional validation role, becomes a mandatory requirement for progress. In order to materialize this synergism, it is imperative that we develop a common ground between simulations and physical experiments. The digital imaging technique has turned flow visualization from a qualitative tool into a powerful flow diagnostic method. This development in digital imaging technology has created a unique and novel link between the development of experimental and computational approaches that interactively help each other in order to develop a better understanding of the physics of turbulent flows which will lead to subsequent development of models for conducting realistic simulation of industrial-type 
flows. For the purpose of discovering new phenomena, experiments remain the most economical tool for fluid mechanics, while simulations, once they have been verified against experiments, are more suitable for producing comprehensive flow databases. Simulations can be used for three-dimensional visualization of flow quantities that the current state-of-the-art flow diagnostic tools are unable to provide.

The computation of physical phenomena without developing a physical understanding can often produce misleading results. On the other hand, misguided experiments can equally contribute to the generation of more questions than answers. These somewhat orthogonal approaches have created two cultures of fluid mechanicians. The continuation of this two-culture mentality, namely experimentalists and numerical analysts who conduct experiments and simulations in isolation, would delay reaching the goals of understanding, modeling and predicting turbulence. To solve this problem, we need to include provisions in our university and government/industrial research policies to accommodate and encourage a new generation of scientists and engineers who understand and appreciate both simulations and experiments.

\section{Acknowledgment}

Anatol Roshko and Anthony Leonard have been my inspiration in developing the points of view in this paper. The encouragement of Edwin Rood has been the key factor in organizing and materializing this paper. Doug Dommermuth, George Karniadakis, Turgut Sarpkaya, Fred Stern, and Dick Yue have been kind enough to provide many clarifying discussions on some of the key issues in CFD. My thanks also goes to Ron Adrian, Nobuhide Kasagi, Dorian Liepmann and Jerry Westerweel for providing data and insight for the latest developments in PIV, DPIV and PTV techniques. Some of the critical comments provided by the reviewers of this paper have been extremely helpful in refining the final manuscript. Last, but not least, I would like to thank Ron Henderson for his critical comments based on his deep enthusiasm for both simulations and experiments.

\section{References}

Adrian, R. J., 1991, "Particle-Imaging Techniques for Experimental Fluid Mechanics," Annual Review of Fluid Mechanics, Vol, 23, pp. 261-304.

Baldwin, W. S., and H. Lomax, 1978, "Thin-Layer Approximate and Algebraic Model for Separated Turbulent Flows," AIAA paper 78-257.

Barnhart, D. H., R. J. Adrian, and G. C. Papen, 1994, "Phase-Conjugate Holographic System for High Resolution PIV," Applied Optics, Vol. 3, No. 30, pp. 7159-7170, October 20.

Batchelor, G. K., 1953, The Theory of Homogeneous Turbulence, Cambridge UP, England.

Brown, G. L., and A. Roshko, 1974, "On Density Effects and Large Structure in Turbulent Mixing Layers," Journal of Fluid Mechanics, Vol. 64, pp. 775816.

Dabiri, D., and M. Gharib, 1991, “Digital Particle Image Thermometry: The Method and Implementation," Experiments in Fluids, Vol. 11, pp. 77-86.

Dimotakis, P. E., 1993, "Some Issues on Turbulent Mixing and Turbulence," GALCIT Report FM 93-1.

Dommermuth, D. G., 1993, "The Laminar Interaction of a Pair Vortex Tube with a Free Surface,' Journal of Fluid Mechanics, Vol. 246, pp. 91-115.

Eggels, J. G. M., F. Unger, M. H. Weiss, J. Westerweel, R. J. Adrian, R. Friedrich, and F. T. M. Nieuwstadt, 1994, "Fully Developed Turbulent Pipe Flow: A Comparison Between Direct Numerical Simulation and Experiment," Joumal of Fluid Mechanics, Vol. 268, pp. 175-209.

Fabris, Drazin, D. Marcus, and D. Liepmann, 1995, "Quantitative Experimental and Numerical Investigation of a Vortex Ring Impinging on a Wall," submitted to Physics of Fluids.

Ferziger, J. H., 1977, "Large Eddy Numerical Simulations of Turbulent Flows," AIAA J., Vol. 15, pp. 1261-1267.

Ferziger, J., 1993, "'Simulation of Complex Turbulent Flows: Recent Advances and Prospects in Wind Engineering," Journal of Wind Engineering and Industrial Aerodynamics, Vols. 46 and 47, pp. 195-212.

Frisch, U., and S. A. Orszag, 1990, "Turbulence-Challenges for Theory and Experiment," Physics Today, Vol. 43, pp. 24-32.

Germano, M., U. Piomellig, P. Moin, and W. H. Cabot, 1991, "A Dynamic Sub-Grid Eddy Viscosity Model," Physics of Fluids A, Vol. 3, No. 7, pp. 17601765 .

Gharib, M., and A. Weigand, 1995, "Experimental Studies of Vortex Disconnection and Connection at a Free Surface," submitted to Journal of Fluid Mechanics.
Gharib, M., and C. Willert, "Particle Tracing: Revisited," (A) AIAA Paper No. AIAA-88-3776-CP, 1935-1943. (B) Chapter 3, Advances in Experimental Fluid Mechanics, Lecture Notes in Engineering, Vol. 37, Springer-Verlag, pp. $109-126,1989$.

Henderson, R., and G. E. Karniadakis, 1995, “Unstructured Spectral Element Methods for Simulation of Turbulent Flows," to appear in Journal of Computational Physics.

Henderson, R., 1994, "Unstructured Spectral Element Methods: Parallel Algorithms and Simulations," Ph.D. thesis, Princeton University.

Hinze, J. O., 1959, Turbulence: An Introduction to Its Mechanism and Theory, McGraw-Hill, New York.

Humphrey, J. A. C., Devarakonda, R., and Queipo, N., 1991, "Interactive Computational-Experimental Methodologies (ICEME for Thermofluids Research: Application to the Optimized Packaging of Heated Electronic Components,' Computer and Computing in Heat Transfer Science and Engineering, KT. Yang and W. Nakayama, eds., CRC Press.

Jimenez, J., 1994, "Resolution Requirements for Velocity Gradients in Turbulence," Center for Turbulence Research Annual Brief, Stanford Univ., AMES Research Center, NASA.

Karniadakis, G. E., and S. Orszag, 1990, “Nodes, Modes and Flow Codes,' Physics Today, American Institute of Physics.

Kasagi, N., and Y. Sata, 1992, "Recent Development in Three-Dimensional Particle Tracking Velocimetry,"' Proceedings of Flow Visualization Conference VI, Yokohama, Japan.

Kim, J., Moin, P., and Moser, R., 1993, "'Turbulence Statistics in Fully Developed Channel Flow at Low Reynolds Number," Journal of Fluid Mechanics, Vol. 255, pp. $65-90$.

Kline, S. J., Reynolds, W. C., Schraub, F. A., and Runstadler, P. W., 1967 "The Structure of Turbulent Boundary Layers," Journal of Fluid Mechanics, Vol. 30, pp. 741-773.

Kolmogorov, A. N., 1941, "The Local Structure of Turbulence in Incompressible Viscous Fluids at Very Large Reynolds Numbers," Dokl. Nauk. SSSR, Vol 30, pp. 301-305, ( see e.g., L. D. Landau and E. M. Lifshitz, Fluid Mechanics, Pergamon, 116-123, 1959).

Koochesfahani, M., and P. Dimotakis, 1980, "Mixing and Chemical Reactions in a Turbulent Liquid Mixing Layer,' $J F M$, Vol. 170.

Launder, B. E., D. P. Tgelepidakis, and B. A. Younis, 1987, "A SecondMoment Closure Study of Rotating Channel Flow," Journal of Fluid Mechanics, Vol. 183 , pp. $63-75$.

Le Penven, L., Gence, J. N., and Comte-Bellot, G., 1985, "On the Approach to Isotropy of Homogenous Turbulence: Effect of the Partition of Kinetic Energy Among the Velocity Components," Frontiers in Fluid Mechanics, Springer-Verlag: New York.

Leonard, A., 1985, "Computing Three-Dimensional Incompressible Flows with Vortex Elements,' Annual Review of Fluid Mechanics, Vol. 17, pp. 523-59.

Leschziner, M. A., 1993, "Computational Modeling of Complex Turbulent Flow: Exceptions, Reality and Prospects," Journal of Wind Engineering and Industrial Aerodyn., Vols. 46 and 47, pp. 37-51.

Lesieur, M., 1989, Turbulence in Fluids, Klewer Academic Publishers (2nd ed. 1991 ).

Liepmann, D., and D. Dommermuth, 1991, "Quantitative Experimental and Numerical Investigation of a Vortex Ring Impinging on a Wall," Proceedings of $A P S / D F D$, Scottsdale, AZ.

Lin, J.-C., and Rockwell, D., 1995, “Transient Structure of Vortex Breakdown on a Delta Wing at Angle of Attachment," AlAA Journal, Vol. 33, No. 1, pp. 612.

Liou, W. W., and P. G. Morris, 1992, "Weakly Nonlinear Models for Turbulen Mixing in a Plane Mixing Layer,' Physics of Fluids A, Vol. 4 (12), pp. 27982808

Lundgren, T. S., 1982, "Strained Spiral Vortex Model for Turbulent Fine Structure," Physics of Fluids, Vol. 25, p. 2193.

Merzkirch, W., 1987, Flow Visualization, Academic Press.

Moin, P., and P. Kim, 1982, "Numerical Investigation of Turbulent Channel

Flow," Journal of Fluid Mechanics, Vol. 118, pp. 341-371.

Murakami, S., Mochida, A., and K. Hiki, 1985, "Three-Dimensional Numerical Simulation of Air Flow Around a Cubic Model by Means of LES,' Journal of Wind Engineering and Industrial Aerodynamics, Vol. 25, pp. 291-305.

Newman, D., and G. E. Karniadakis, 1995, "Simulations and Models of Flow over a Flexible Cable: Standing Wave Patterns,' ' to be presented at ASME/JSME Fluids Engineering Conf., Hilton Head, SC, Aug.

Pearlstein, A. J., and B. Carpenter, 1995, " On the Determination of Solenoidal or Compressible Velocity Fields From Measurements of Passive and Reactive Scalars," Physics of Fluids, Vol. 7, No. 4, pp. 754-763.

Pullin, D., and P. G. Saffman, 1993, "On the Lundgren-Townsend Model of Turbulent Fine Scales," Physics of Fluids A, Vol. 5 (1), pp. 126-145.

Raffel, M., M. Gharib, O. Ronneberger, and J. Kompenhans, 1995, "Feasibility Study of Three-Dimensional PIV by Correlating Images of Particles Within Parallel Light Sheet Planes," accepted in Experiments in Fluids.

Rizzi, A., and Engquist, B., 1987, "Selected Topics in the Theory and Practice of Computational Fluid Dynamics,' Journal of Computational Physics, Vol. 72 No. 1, Sept.

Rodi, W., 1993, “On the Simulation of Turbulent Flow Past Bluff Bodies," Journal of Engineering and Industrial Aerodynamics, Vols. 46 and 47, pp. 3-9. Rogallo, and P. Moin, 1984, "Numerical Simulation of Turbulent Flows," Annual Review of Fluid Mechanics, Vol. 16, pp. 99-137.

Roshko, A., 1992, "Instability and Turbulence in Shear Flows," Theoretical and Applied Mechanics. 
Saddoughi, S. G., and S. V. Veeravalli, 1994, "Local Isotropy in Turbulent Boundary Layers at High Reynolds Numbers," Journal of Fluid Mechanics, Vol. 268, pp. 333-372

Sarpkaya, T., and Suthon, P., 1991, "Interaction of a Vortex Couple with a Free Surface," Experiments in Fluids, Vol. 11, pp. 205-217.

Sarpkaya, T., 1988, "Computational Methods with Vortices: The 1988 Freeman Scholar Lecture,” ASME Journal of Fluids ENGINEERING, Vol. 111, pp. 5-52. Sarpkaya, T., M. Magee, and C. Merril, 1994, "Vortices, Free Surface and Turbulence," Proceedings of ASME Fluids Engineering Division Summer Meeting, Lake Tahoe, Nevada, June 19-23, RED-Vol. 181

Singh, A., 1991, “Optic Flow Computation,"' IEEE, Computer Society Press. Speziale, C. G., 1990, "Discussion of Turbulence Modeling: Past and Future," Lecture Notes in Physics, ed. J. L. Lumley, Vol. 357, Springer-Verlag: New York, pp. 354-368.

Tahra, Y., and F. Stern, 1994, "Validation of an Interactive Approach for Calculating Ship Boundary Layers and Wakes for Nonzero Frouhe Numbers," Journal of Computers and Fluids, Vol. 23, No. 6, pp. 785-816.

Visbal, M. R., 1994, "Onset of Vortex Breakdown Above a Pitching Delta

Wing," AIAA Journal, Vol. 32, pp. 1568-1575.

Westerweel, J., A. A. Draad, J. G. van der Hoeven, and J. van Oord, 1995, "Measurement of Fully-Developed Turbulent Pipe Flow with Digital Particle Image Velocimetry," submitted to Exp. Fluids.

Westerweel, J., 1993, Digital lmage Velocimetry: Theory and Application, Delft UP, Delft, Netherlands.

Willert, C., and M. Gharib, 1991, "Digital Particle Image Velocimetry,' Experiments in Fluids, Vol. 10, pp. 181-183.

Zhang, C., and D. Yue, 1995, private communication.

Zhang, X., and C. S. Cox, 1994, "Measuring the Two-Dimensional Structure of a Wavy Water Surface Optically: A Surface Gradient Detector,' Experiments in Fluids, Vol. 17, pp. 225-237

Zhang, X., D. Dabiri, and M. Gharib, 1996, “Optical Mapping of Fluid Density Interfaces: Concepts and Implementation," Review of Scientific Instruments., May, Vol. 67

\section{A P P E N D I X}

When one starts talking about turbulence, one gets pushed immediately into a statistical way of thinking - what is the mean velocity, what are the r.m.s. turbulence fluctuations, how large are the Reynolds stresses? Statistical quantities come from long averages relative to some fundamental time scale of the flow, so it seems like an interesting question to ask what the computational time scale is to compute a turbulent flow versus the laboratory time scale to generate one? Consider this for one of the classic problems in bluff body wakes: flow past a circular cylinder.
The wake of a circular cylinder becomes "turbulent" at a relatively low Reynolds number $\operatorname{Re}=U d / v$ of a few hundred. It is fully turbulent at $\mathrm{Re}=1000$ but still accessible to direct numerical simulations because the range from $O(d)$ to the dissipation scale is fairly narrow, say three to four decades in an energy scale based on the kinetic energy per unit volume of the mean flow. This is an ideal case for comparing simulations and experiments because both should be well within our current capabilities.

First, consider the numbers for the experiment. Assume the working fluid is air $\left(v=1.5 \times 10^{-5} \mathrm{~ms} / \mathrm{s}\right)$, and the tunnel velocity is a steady $5 \mathrm{~m} / \mathrm{s}$. A cylinder with diameter $d=2.5$ mm can be made from brass and supported to prevent vibrations, giving a value for $\operatorname{Re}$ that is $O(1000)$. We know the dimensionless shedding frequency or Strouhal number is $\mathrm{St} \approx 0.2$, so the dimensional shedding frequency is $f=\mathrm{St} U / d=400 \mathrm{~Hz}$. In the wind tunnel, the fundamental time scale for the cylinder wake is 0.0025 seconds.

What about a simulation of the same flow? Consider a stateof-the-art calculation with a highly accurate multi-domain spectral method. On a 64 processor Intel Paragon with a sustained rate of 0.5 Gflops, such a calculation can integrate a model with $O\left(10^{6}\right)$ degrees of freedom ("mesh points") as fast as 5 seconds per time step. Moving up the scale, that is 3 hours per shedding cycle (2100 time steps); 4 shedding cycles per day (this is a shared facility, so you can only count on 12 hours of dedicated time); 25 shedding cycles per week; or 100 shedding cycles in a month. Of course, that is a month of "real time" as opposed to a month of computer time, but it is an accurate picture of what might be expected at a national supercomputing center. Here is the bottom line: one month of high-performance computer-simulated flow equals 0.25 seconds of experimentation time in the wind tunnel, for this case. Even assuming we have a dedicated facility, it places the ratio of time scales at roughly 4,000,000:1. It is important to keep in mind that simulations provide comprehensive flow information (e.g., unsteady pressure field) which might not be attained through experiments. 